\title{
Lowering Outage Probability in ad hoc Networks by Nearest Neighbor FDMA Scheduling
}

\author{
Ralph Tanbourgi, Jens P. Elsner and Friedrich K. Jondral \\ Karlsruhe Institute of Technology, Germany \\ $\{$ ralph.tanbourgi, jens.elsner, friedrich.jondral\}@kit.edu
}

\begin{abstract}
This paper investigates the performance of a wireless ad hoc network capable of dynamically coordinating a set of orthogonal frequency channels such that every receiver and its nearest interferer operate in different channels. The distribution of the ratio of the interference powers of the second and first interferer is derived to show that the nearest interferer has dominant character, particularly in the absence of fading. Lower and upper bounds on outage probability are derived for the path loss model. For Rayleigh fading, an approximation is presented. Simulation results show that nearest neighbor scheduling is beneficial, thereby avoiding interference effectively. In contrast to other techniques such as successive interference cancellation, the complexity of nearest neighbor scheduling remains comparatively low.
\end{abstract}

\section{INTRODUCTION}

A wireless ad hoc network consists of a collection of nodes communicating without the help of any predefined infrastructure. Ad hoc networks have attracted much interest in the communication community due to steady advances in the design of low-cost hardware and the emergence of infrastructureless applications for mobile, vehicular and sensor networks.

In a recent debate [1], the use of spread-spectrum techniques, i.e., direct sequence code division multiple access (DS-CDMA) and frequency hopping CDMA (FH-CDMA), in ad hoc networks was investigated and their advantages and disadvantages over narrowband systems were highlighted. It was found that FH-CDMA is superior to both narrowband and DS system in terms of transmission capacity due to its interference avoidance nature. As a result, FH-CDMA allows longer hops to be taken at the same outage probability which in turn may improve energy efficiency and decrease end-toend delay variance.

In contrast, DS-CDMA in conjunction with multi-user techniques such as successive interference cancellation (SIC) can also improve system performance as presented in [2], [3], though its implementation in ad hoc networks may be impracticable: the performance of SIC is strongly limited by the accuracy of channel estimation [1], which may also have an adverse effect on performance if channel estimation is inaccurate. Delay-sensitive services may be impossible due to decoding and processing time. Moreover, the computational complexity may not be justified in energy-limited ad hoc networks.

In the case of FH-CDMA and narrowband systems, Jindal et al. [4] obtained the optimum bandwidth split, i.e., the optimal number of channels, which is an increasing function of the path loss exponent $\alpha$ and of the ratio of operating bandwidth to transmission rate. For practical networks, this number is expected to be high for the following reasons:

- High transmission bandwidth introduces increased hardware complexity and possibly expensive devices. Hence, transmission bandwidth is likely to be small compared to the operating bandwidth.

- Ad hoc networks will often be found in mobile applications and will be exposed to rural or even urban channel conditions: in this case, the path loss exponent $\alpha$ is high.

This observation motivates the analysis of scheduled networks, in which a large set of channels can be used to successfully coordinate transmissions to increase link performance. The resulting model can be thought of as an FH-CDMA scheme with the additional feature that every receiver and its associated nearest interferer are assigned orthogonal hopping sequences. The interference avoidance feature of FH-CDMA is hence increased by assuring that the nearest (and potentially strongest $^{1}$ ) interferer is orthogonalized.

There are two main reasons for following the idea of orthogonalizing only the nearest interferer rather than trying to cancel out more: first, coloring a nearest neighbor graph has a low complexity since the number of edges is comparatively small. In conjunction with decentralized fast coloring algorithms, an ad hoc network capable of dynamically orthogonalizing each node's nearest interferer is thus feasible and practical. Second, it is often sufficient to orthogonalize only the nearest interferer to obtain a significant performance gain since the nearest interferer has a dominant effect on the aggregated interference at the receiver [5]. Outage probability is often taken as a measure to quantify link performance in stochastic environments where communication is subject to, e.g., multi path fading and interference from randomly located nodes. Besides, this quantity is strongly connected to the packet loss rate, which can be used as a quality of service indicator in admission control mechanisms to efficiently allocate resources and manage routing related tasks in (mobile) ad hoc networks [6].

The remainder of the paper is organized as follows: First, the network model is presented in Section II where we shortly summarize prior results on which this work is built.

\footnotetext{
${ }^{1}$ This is true if nodes transmit with same power and the path loss model is assumed. In case of fading, the nearest interferer is the strongest on average.
} 
In Section III, we first analyze the distribution of the ratio of the interference powers of the second and the first interferer. Then, we introduce the nearest neighbor scheduling model and derive lower and upper bounds on outage probability for the path loss model and an approximation for Rayleigh fading. Section IV presents some simulation results in order to validate the analysis. Section V concludes the paper.

\section{System ModeL}

We assume an infinite collection of nodes spread over the plane $\mathbb{R}^{2}$ and forming a wireless ad hoc network. The transmitters $\left\{X_{i}\right\}$ are uniformly and independently distributed according to a homogeneous Poisson point process (PPP) $\Pi(\lambda)$, where $\lambda$ is the density of transmitters. $X_{i}$ denotes the $i$-th transmitter as well as its location on $\mathbb{R}^{2}$. Every transmitter $X_{i}$ communicates with an associated receiver $X_{i}^{\mathrm{rx}}$ situated at most $r$ units away. According to Slivnyak's theorem [7], the distribution of $\Pi(\lambda)$ is not affected by the addition of a specific point. Thus, we can place a reference receiver in the origin and an associated reference transmitter $r$ units away. In the following, the PPP $\Pi(\lambda)$ is considered from the viewpoint of the reference receiver. As we consider only interferencelimited networks, the effect of thermal noise is neglected. Without loss of generality, all transmitters are assumed to transmit with unit power. Furthermore, interference at the receiver is treated as white noise and the total bandwidth available for transmission is $B \mathrm{~Hz}$. Assuming a path loss function of the form $\left|X_{i}-X_{j}\right|^{-\alpha}$, where $\alpha>2$ is the path loss exponent, the outage probability is defined as [5]

$q(\lambda) \triangleq \mathbb{P}\left\{\frac{r^{-\alpha}}{\sum_{i \in \Pi(\lambda)}\left|X_{i}\right|^{-\alpha}}<\beta\right\}=\mathbb{P}\left\{Y(\lambda)>r^{-\alpha} \beta^{-1}\right\}$,

where the shot-noise process $Y(\lambda) \triangleq \sum_{i \in \Pi(\lambda)}\left|X_{i}\right|^{-\alpha}$ is the aggregate interference power at the reference receiver and $\beta \triangleq 2^{\frac{R}{B}}-1$ is the required signal-to-interference ratio (SIR) threshold for supporting a rate $R$.

The total bandwidth $B$ is further divided into $M$ orthogonal channels. In the non-scheduling FH-CDMA case, each transmitter-receiver pair independently chooses a channel $M_{j}$, where $j=1, \ldots, M$ with probability $\mathbb{P}\left\{M_{j}\right\}=\frac{1}{M}$, for communication. Then, the collection of transmitters interfering at the reference receiver forms an independently marked version of the PPP $\Pi(\lambda)$, denoted by $\tilde{\Pi}\left(\lambda_{M}\right)$, where $\lambda_{M}=\frac{\lambda}{M}$ is the density of transmitters in one channel. The outage probability $q(\lambda)$ now depends on the value of $M$ and is given by

$$
\begin{aligned}
q(\lambda) & =\mathbb{P}\left\{\frac{r^{-\alpha}}{\sum_{i \in \tilde{\Pi}\left(\lambda_{M}\right)}\left|X_{i}\right|^{-\alpha}}<\beta\right\} \\
& =\mathbb{P}\left\{\tilde{Y}>r^{-\alpha} \beta^{-1}\right\} .
\end{aligned}
$$

Note that $\beta=2^{\frac{R}{B}} M-1$ is now an increasing function of the number of channels $M$ : better channel conditions are required since less transmission bandwidth is now available while the supported transmission rate is still $R$. In [4], Jindal et al. showed that the optimal $M^{*}$ that minimizes (2) is given by: ${ }^{2}$

$$
M^{*}=\frac{B}{R} \log _{2}\left(\exp \left(\frac{\alpha}{2}+\mathcal{W}\left(-\frac{\alpha}{2} e^{-\frac{\alpha}{2}}\right)\right)\right),
$$

where $\mathcal{W}(z)$ is the principle branch of the Lambert $\mathcal{W}$ function solving $\mathcal{W}(z) e^{\mathcal{W}(z)}=z$.

In the following we will always assume that $M=M^{*}$ and that this number is sufficiently large such that nearest neighbor scheduling is feasible. Note that the assumption $M=M^{*}$ does not yield the optimal $M$ minimizing $q(\lambda)$ for our scheduling scheme in general, but allows for fair comparison to the nonscheduled case in terms of hardware complexity (filter, ADC, etc.). In [8], the optimal $M^{*}$ minimizing outage probability for a full scheduled FDMA network is studied.

\section{Nearest Neighbor Scheduling}

We assume that nodes are able to coordinate their frequency channel assignments. This coordination process takes places between local nodes such that every receiver and its nearest interferer within the scheduling range are assigned different channels. The scheduling process is performed for a predefined time period prior to transmission, e.g., by exchanging information on a distributed control channel or by periodically transmitting a pilot signal on the chosen channel. In the latter case, no information is transmitted and synchronization is not necessary whereas in the former case, the information content is low which allows for channel coding with a small coding rate. Therefore, we assume that the scheduling range exceeds the (data) communication range $r$, but remains less than $r_{s}=r \beta^{\frac{1}{\alpha}}$, denoting the radius of the near interference region around a receiver. In this region the presence of already one interferer leads to an outage at the receiver in the absence of fading (cf. [5]). We assume higher-order modulation, i.e., $\beta>1$, so $r_{s} \geq r$ and the near interference region exceeds the communication region.

Limiting the scheduling range is reasonable for the following reasons: first, it might not be practical to allow an unconstrained scheduling range since this imposes an undesirable decoding delay at the receivers and hence increases the termination time of the scheduling algorithm. Second, it is often sufficient to avoid nodes within the near interference region only since they have dominant character.

In a graph theoretical context, we have a coloring problem of a graph $\mathcal{G}$ with vertices $\left\{\left\{X_{i}\right\} \cup\left\{X_{i}^{\mathrm{rx}}\right\}\right\}$ and the set of directed edges $\mathcal{E}=\left\{\left\{e_{i j}\right\} \mid j=\arg \min _{n \neq i}\left\{\left|X_{n}-X_{i}^{\mathrm{rx}}\right|\right\}\right\}$, i.e., a modified Euclidean nearest neighbor graph on $\mathbb{R}^{2}$. Here, the presence of an edge $e_{i j}$ indicates that transmitter $X_{j}$ is the nearest interferer of receiver $X_{i}^{\mathrm{rx}}$ and hence means that the choice of $X_{j}, X_{j}^{\mathrm{rx}}$ of selecting a certain channel is constrained by the choice of $X_{i}, X_{i}^{\mathrm{rx}}$ and vice-versa. We denote by $n_{k}\left(X_{i}^{\mathrm{rx}}\right) \in \Pi(\lambda)$ the $k$-th nearest interfering transmitter of receiver $X_{i}^{\text {rx }}$. In particular, $n_{k}(0)$ refers to the $k$-nearest interferer of the reference receiver (located in the origin).

\footnotetext{
${ }^{2}$ Note that we implicitly assume here that every node transmits with rate $R$ on average.
} 


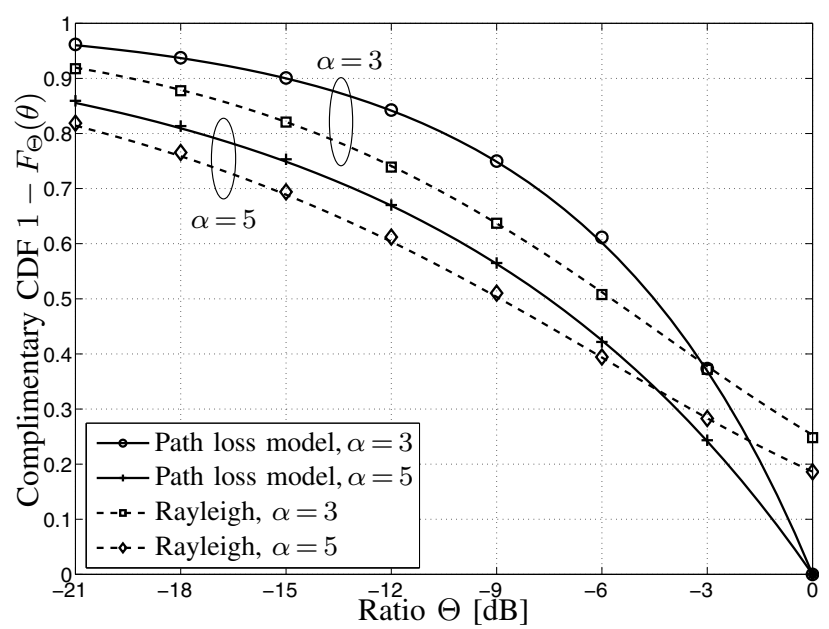

Fig. 1. Complementary CDF $1-F_{\Theta}(\theta)$ for the path loss model and for the Rayleigh fading model. Empirical complimentary CDF is indicated by the marks.

\section{A. Distribution of the ratio of the interference powers of the second and the first interferer}

To understand why it is already beneficial to orthogonalize only the nearest interferer, we will investigate the distribution of $\Theta$ denoting the ratio of the (instantaneous) received interference powers of the second and the first interferer. If $\Theta$ is small the received interference power from the interferers $n_{k}\left(X_{i}^{\mathrm{rx}}\right)$, $k=2,3, \ldots$, are negligible since interferer $n_{1}\left(X_{i}^{\mathrm{rx}}\right)$ creates the heaviest interference, although not necessarily exceeding the total aggregated interference power from all other nodes. However, for moderate to high $\alpha$, the received interference power from the nearest interferer is expected to have dominant character, which can be analyzed by means of the distribution of $\Theta$.

Theorem 1. The distribution of $\Theta$ : Let $\left|n_{1}\left(X_{i}^{\mathrm{rx}}\right)-X_{i}^{\mathrm{rx}}\right|^{-\alpha}$ and $\left|n_{2}\left(X_{i}^{\mathrm{rx}}\right)-X_{i}^{\mathrm{rx}}\right|^{-\alpha}$ be the interference power of the first and the second interferer, measured at the receiver $X_{i}^{\mathrm{rx}}$. Then, the random variable $\Theta \triangleq \frac{\left|n_{2}\left(X_{i}^{\mathrm{rx}}\right)-X_{i}^{\mathrm{rx}}\right|^{-\alpha}}{\left|n_{1}\left(X_{i}^{\mathrm{rx}}\right)-X_{i}^{\mathrm{rx}}\right|^{-\alpha}}$ has distribution

$$
F_{\Theta}(\theta)=\theta^{\frac{2}{\alpha}}, \quad 0 \leq \theta \leq 1 .
$$

In the case of Rayleigh fading, the corresponding ratio $\Theta_{\mathrm{Ray}} \triangleq \frac{G_{k}\left|n_{2}\left(X_{i}^{\mathrm{rx}}\right)-X_{i}^{\mathrm{rx}}\right|^{-\alpha}}{G_{\ell}\left|n_{1}\left(X_{i}^{\mathrm{rx}}\right)-X_{i}^{\mathrm{rx}}\right|^{-\alpha}}$, where $G_{k}, G_{\ell}$ are independent unit-mean exponential random variables, is distributed as

$$
F_{\Theta_{\text {Ray }}}\left(\theta_{\text {Ray }}\right)=F\left(1, \frac{2}{\alpha}, \frac{2}{\alpha}+1,-\frac{1}{\theta_{\text {Ray }}}\right), \quad \theta_{\text {Ray }} \geq 0,
$$

where $F(a, b, c, z)=\sum_{s=0}^{\infty} \frac{(a)_{s}(b)_{s}}{(c)_{s} s !} z^{s}$ is the hypergeometric function.

A proof of Theorem 1 is given in Appendix A.

Fig. 1 shows the complementary cumulative density function (CDF) of $\Theta$ for both the path loss model and the Rayleigh fading model. For the path loss model, we can see that if $\alpha \geq 3$, the probability $\mathbb{P}\{\Theta \geq 0.5\}$ is less than 0.4. In particular, for $\alpha=5, \mathbb{P}\{\Theta \geq 0.5\}=0.25$, which underlines the fact that scheduling more than the nearest interferer may not yield a significant improvement. This, however, can not be observed in the case of Rayleigh fading since the received interference power of the second interferer may exceed the interference power of the nearest. Also, the mean of $\Theta_{\text {Ray }}$ does not exist since the ratio $\frac{G_{k}}{G_{\ell}}$ has a heavy-tailed distribution. In case of the path loss model, the mean can be calculated as $\mathbb{E}\{\Theta\}=\frac{2}{2+\alpha}$. Another interesting fact is that the distribution of both $\Theta$ and $\Theta_{\text {Ray }}$ is independent of $\lambda$.

\section{B. Outage Probability: Path loss Model}

Following the notation in [5], we decompose the outage event into two disjoint events, i.e., outage due to the near interference region $\left(\tilde{Y}^{\mathrm{n}}>r^{-\alpha} \beta^{-1}\right)$ and outage due to the far interference region $\left(\tilde{Y}^{\mathrm{f}}>r^{-\alpha} \beta^{-1}\right)$. In the near interference region, the presence of at least one transmitter (after having removed the nearest one), transmitting in the same channel is a sufficient condition for outage at the reference receiver. This event can therefore be written as $\left\{\tilde{Y}^{\mathrm{n}}>r^{-\alpha} \beta^{-1}\right\}=\left\{\left(\tilde{\Pi}\left(\lambda_{M}\right) \backslash n_{1}(0)\right) \cap d\left(0, r_{s}\right) \neq \emptyset\right\}$, where $d\left(0, r_{s}\right)$ is a disc of radius $r_{s}=r \beta^{\frac{1}{\alpha}}$ denoting the near interference region, centered around the reference receiver. In the far interference region $\bar{d}\left(0, r_{s}\right)$, the aggregated interference from all nodes transmitting in the same channel must exceed $r^{-\alpha} \beta^{-1}$, i.e., $\tilde{Y}^{\mathrm{f}}>r^{-\alpha} \beta^{-1}$, in order to create outage. Hence, we can write ${ }^{3}$

$$
\begin{gathered}
q(\lambda)=\mathbb{P}\left\{\left(\tilde{\Pi}\left(\lambda_{M}\right) \backslash n_{1}(0)\right) \cap d\left(0, r_{s}\right) \neq \emptyset\right\}+ \\
\left(1-\mathbb{P}\left\{\left(\tilde{\Pi}\left(\lambda_{M}\right) \backslash n_{1}(0)\right) \cap\left(d\left(0, r_{s}\right) \neq \emptyset\right)\right\}\right) \\
\quad \times \mathbb{P}\left\{\tilde{Y}^{\mathrm{f}}>r^{-\alpha} \beta^{-1}\right\} .
\end{gathered}
$$

Theorem 2. Bounds on the outage probability $q(\lambda)$ : Let the number of channels $M$ be sufficiently large such that each receiver and its nearest interferer separated by at most $r_{s}=r \beta^{\frac{1}{\alpha}}$ can operate on different channels. Further define the function

$$
\phi(\lambda, x) \triangleq e^{-\mu} \sum_{k=2}^{\infty} \frac{\mu^{k}}{k !}\left(1-(1-x)^{k-1}\right),
$$

where $\mu=\lambda \pi r^{2} \beta^{\frac{2}{\alpha}}$. Then, the outage probability $q(\lambda)$ is lower bounded by

$$
q^{l}(\lambda)=\phi(\lambda, a)
$$

where $a=\frac{4\left(1+\beta^{1 / \alpha}\right)}{M\left(2+\beta^{1 / \alpha}\right)^{2}}$ and upper bounded by

$q^{u}(\lambda)=\phi\left(\lambda, \frac{1}{M}\right)+\frac{\mu(1-\phi(\lambda, a))}{M(\alpha-1)}\left(1-\frac{2 \mu}{M(\alpha-2)}\right)^{-2}$.

A proof of Theorem 2 is given in Appendix B.

${ }^{3}$ Note that the third outage event $\left\{\tilde{Y}^{\mathrm{n}}+\tilde{Y}^{\mathrm{f}}>r^{-\alpha} \beta^{-1} \mid \tilde{Y}^{\mathrm{n}}<r^{-\alpha} \beta^{-1} \cap\right.$ $\left.\tilde{Y}^{\mathrm{f}}<r^{-\alpha} \beta^{-1}\right\}$ has zero probability, since $\left\{\tilde{Y}^{\mathrm{n}}<r^{-\alpha} \beta^{-1}\right\}$ implies that $\left\{\left(\tilde{\Pi}\left(\lambda_{M}\right) \backslash n_{1}(0)\right) \cap d\left(0, r_{s}\right)=\emptyset\right\}$ and hence $\tilde{Y}^{\mathrm{n}}=0$ (cf. [5]). 


\section{Outage Probability: Rayleigh Fading}

In the case where the signals are subject to Rayleigh fading, the aggregated interference at the reference receiver can be written as

$$
\tilde{Y} \triangleq \sum_{i \in \tilde{\Pi}\left(\lambda_{M}\right)} G_{i}\left|X_{i}\right|^{-\alpha},
$$

where the channel gains $G_{i}$ are assumed to be i.i.d. according to a unit-mean exponential distribution. The corresponding outage probability is then given by [9]

$$
q(\lambda) \triangleq \mathbb{E}\left\{\mathbb{P}\left\{G<\tilde{Y} r^{\alpha} \beta \mid \tilde{Y}\right\}\right\} .
$$

We still assume that every receiver and its nearest interferer operate on different channels. Unlike in the path loss model, the nearest interferer now might not be the strongest interferer since the associated channel gain at the reference receiver may be small compared to the gains of the second or third nearest interferer. Scheduling the strongest interferer based on the knowledge of the channels gains $G_{i}$ is impractical since the channel coherence time will typically be much smaller than the time needed for proper scheduling.

We further assume that nodes are now able to coordinate frequency channels with nodes that lie beyond $r_{s}$. This assumption is reasonable: if the time period available for the scheduling process is much larger than the channel coherence time, it is likely that high channel gains will appear during this period.

Theorem 3. Approximation of the outage probability $q(\lambda)$ : Let the number of channels $M$ be sufficiently large such that every receiver and its nearest interferer can operate on different channels. Then the outage probability $q(\lambda)$ can be approximated by

$$
\begin{aligned}
q(\lambda) \approx & \tilde{q}(\lambda)=1-\exp \left\{\frac { \lambda \pi } { M } \left(\frac{1}{4 \lambda}\left(1-\frac{1}{1+r^{\alpha} \beta(4 \lambda)^{\frac{\alpha}{2}}}\right)\right.\right. \\
& -r^{2} \beta^{\frac{2}{\alpha}} \Gamma\left(1-\frac{2}{\alpha}\right) \Gamma\left(1+\frac{2}{\alpha}\right) \\
& \left.\left.+r^{2} \beta^{\frac{2}{\alpha}} \mathbb{E}\left\{G^{\frac{2}{\alpha}} \Gamma\left(1-\frac{2}{\alpha}, G r^{\alpha} \beta(4 \lambda)^{\frac{\alpha}{2}}\right)\right\}\right)\right\} .
\end{aligned}
$$

In particular, $\tilde{q}(\lambda)$ is a lower (upper) bound on $q(\lambda)$ if $\lambda<\lambda_{s}$ $\left(\lambda>\lambda_{s}\right)$, where $\lambda_{s}$ denotes the inflection point of $\tilde{q}(\lambda)$, i.e., the solution of the equation $\tilde{q}^{\prime \prime}(\lambda)=0$.

A proof of Theorem 3 is given in Appendix C.

TABLE I

SimUlation RESULTS OF THE CFL ALGORITHM

\begin{tabular}{|c||c|c||c|c|c|}
\hline$\lambda$ & $\mu$ & $\sigma$ & 1 iter & 2 iter & 3 iter \\
\hline $10^{-4}$ & 1.75 & 0.51 & .9950 & .9998 & 1 \\
$10^{-3}$ & 2.37 & 0.52 & .9696 & .9986 & .9999 \\
$10^{-2}$ & 2.56 & 0.57 & .9569 & .9978 & .9998 \\
$10^{-1}$ & 2.63 & 0.60 & .9543 & .9975 & .9998 \\
\hline
\end{tabular}

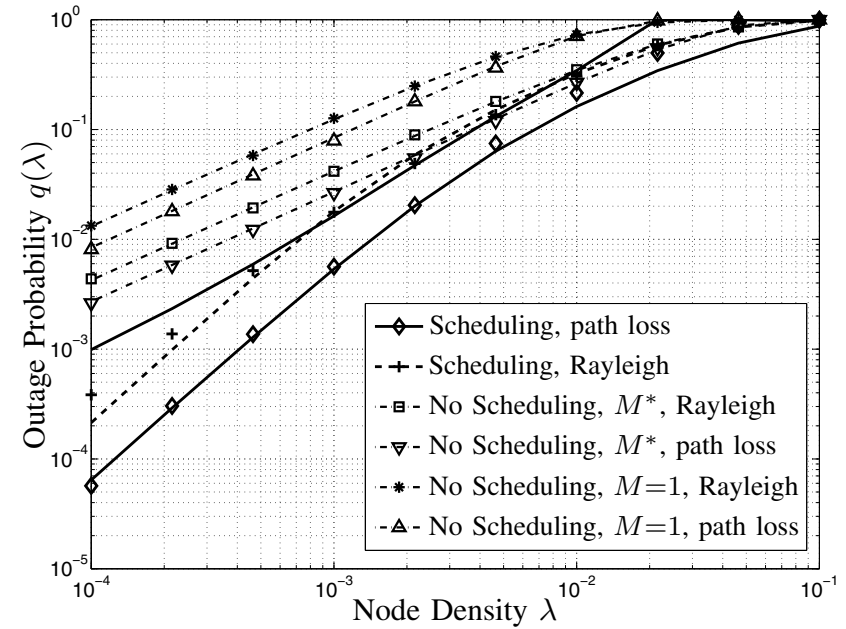

Fig. 2. Outage probability $q(\lambda)$ vs. node density $\lambda$. Theoretical and simulation results are indicated by lines and marks, respectively. For nearest neighbor scheduling, the lower and upper bound from Theorem 2 (path loss model) and the approximation from Theorem 3 (Rayleigh fading) are shown.

\section{Numerical Results}

We use the stochastic coloring algorithm called communication-free learning (CFL) from [10] for modeling the channel assignment problem. The CFL algorithm has some nice properties such as low complexity and fast convergence. In this algorithm, every node randomly selects a channel according to a distribution that depends on the observed channel assignments of the neighbors.

Table I shows the performance of the CFL algorithm with tuning parameter 0.5 for coloring a network (graph $\mathcal{G}$ ) with 500 nodes uniformly distributed in the plane. The number of channels was set to $M=33$. The results were obtained through Monte-Carlo simulations with $10^{4}$ runs. The parameters $\mu, \sigma$ are the mean and the standard deviation of the number of iterations needed for proper coloring of the network. The other columns show the average fraction of successfully scheduled nodes after 1, 2 and 3 iterations.

We conducted further simulations with path loss exponent $\alpha=4, \frac{R}{B}=0.1$ and transmission range $r=10$. For each realization, a PPP was generated on a bounded window $A$ by simulating a Binomial point process with $K$ nodes, where $K \sim \operatorname{Pois}(\lambda|A|),|A|=\bar{K} / \lambda$ and $\bar{K}=500$ was the average number of nodes on $A$, cf. [7]. Fig. 2 shows the performance of nearest neighbor scheduling in terms of outage probability $q(\lambda)$ vs. the node density $\lambda$ for both the path loss model and Rayleigh fading. The performance of non-scheduled systems with no bandwidth partitioning $(M=1)$ and optimal FHCDMA $\left(M=M^{*}\right)$ are also plotted for comparison and validation of the simulation results. It can be seen that the benefit of the proposed scheduling strategy is high, in particular in the low outage probability regime $q(\lambda) \rightarrow 0$. In the region $q(\lambda) \rightarrow 1$, the performance of nearest neighbor scheduling becomes similar to that of non-scheduled optimal FH-CDMA. Furthermore, we observe that the lower bound $q^{l}(\lambda)$ in (8) of Theorem 2 is tight resulting from the dominant interferers of the near interference region, cf. [5]. 


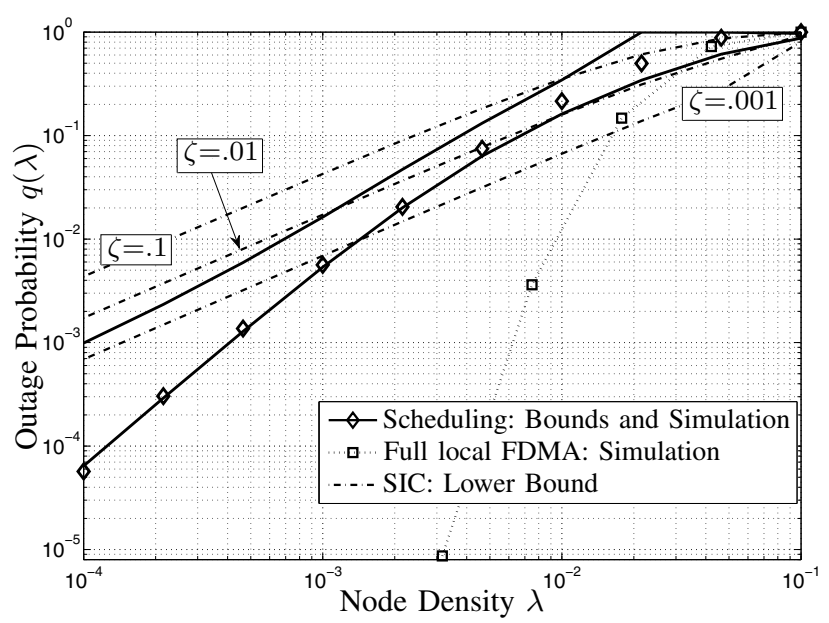

Fig. 3. Outage probability $q(\lambda)$ vs. node density $\lambda$ for nearest neighbor scheduling, full local FDMA scheduling and successive interference cancellation with different $\zeta$.

Fig. 3 shows a comparison between nearest neighbor scheduling and SIC and full local FDMA scheduling, where nodes are capable of orthogonalizing all nodes within the communication region $r$ (cf. [8]). The parameters are the same as above. In case of SIC, the lower bound on outage probability from [2, eq. (48)] with different canceling effectiveness $\zeta$ is shown. We can see that the performance of SIC is rather poor compared to nearest neighbor scheduling, in particular in the low outage probability regime. SIC does not become superior until $\zeta$ becomes sufficiently small and the network is driven in the regime $q(\lambda) \rightarrow 1$. This is explained by the fact that the performance of SIC is constrained by the cancellation effectiveness $\zeta$ [1]. In case of full local FDMA scheduling, outage probability remains low compared to nearest neighbor scheduling since all interferers within the communication range $r$ are now orthogonalized. This benefit, however, comes along with an increase in complexity: the average required number of iterations is $\mu \approx 8.5$. Such an increase may not be practical in ad hoc networks in some cases. Increasing the number of transmitters that can be orthogonalized, e.g., second and third nearest neighbor, changes the slope of $q(\lambda)$ but requires both more orthogonal channels $M$ and more iterations, with full local FDMA scheduling representing the extreme case. Within this trade-off, nearest neighbor FDMA has the lowest complexity.

\section{CONCLUSION}

We analyzed the performance of an FDMA based ad hoc network capable of dynamically orthogonalizing the nearest and potentially strongest interferer. In the case where the number of channels is high, the assumption of successful orthogonalization is reasonable. Orthogonalizing only the nearest interferer is beneficial for two reasons: First, scheduling complexity is kept low and fast convergence of distributed coloring algorithms is ensured. Second, it is often sufficient to orthogonalize the nearest interferer to improve performance significantly. We therefore analyzed the distribution of the ratio of the interference powers of the second and the first interferer for both the path loss and the Rayleigh fading model. For the path loss model, the distribution of the ratio has a power-law character whereas in the case of Rayleigh fading, the distribution is given by a hypergeometric function. It was found that the ratio may be small, in particular for the path loss model. Motivated by this observation, we derived lower and upper bounds on outage probability for the path loss model as well as an approximation of outage probability for Rayleigh fading. The lower bound is tight which is a result of the effect of dominant interferers. Simulations were conducted based on the communication-free learning (CFL) algorithm proposed in [10] for the channel assignment problem in order to validate the theoretical results. It was found that nearest neighbor scheduling increases system performance significantly compared to the non-scheduling case, in particular for low target outage probabilities. In this case, nearest neighbor scheduling is also superior to imperfect successive interference cancellation. The complexity of nearest neighbor scheduling is scalable and remains comparatively low since this scheduling strategy aims at orthogonalizing only the nearest node.

\section{APPENDIX A PROOF OF THEOREM 1}

We first compute the distribution of the auxiliary random variable $Z \triangleq \frac{\left|n_{2}\left(X_{i}^{\mathrm{rx}}\right)-X_{i}^{\mathrm{rx}}\right|}{\left|n_{1}\left(X_{i}^{\mathrm{rx}}\right)-X_{i}^{\mathrm{rr}}\right|}=\frac{R_{2}}{R_{1}}$ as follows:

$$
\begin{aligned}
\mathbb{P}\{Z \leq z\} & =\mathbb{P}\left\{R_{2} \leq z R_{1}\right\} \\
& =\int_{0}^{\infty} F_{R_{2} \mid R_{1}}\left(z r_{1} \mid r_{1}\right) f_{R_{1}}\left(r_{1}\right) \mathrm{d} r_{1} .
\end{aligned}
$$

It is well-known [11] that the random variable $R_{1}$ is Rayleigh distributed with parameter $(2 \lambda \pi)^{-1 / 2}$ and given $R_{1}=r_{1}$, $F_{R_{2} \mid R_{1}}\left(z r_{1} \mid r_{1}\right)=\exp \left\{-\lambda \pi\left(z^{2} r_{1}^{2}-r_{1}^{2}\right)\right\}$ is the probability of no transmitter being in the annulus with radii $r_{1}$ and $z r_{1}$. Hence, (A.1) is computed as

$$
\mathbb{P}\{Z \leq z\}=\int_{0}^{\infty} e^{-\lambda \pi r_{1}^{2}\left(z^{2}-1\right)} 2 \lambda \pi r_{1} e^{-\lambda \pi r_{1}^{2}} \mathrm{~d} r_{1}=z^{-2} .
$$

With the substitution $\Theta=Z^{-\alpha}$, we then finally obtain $F_{\Theta}(\theta)=F_{Z}\left(z^{-\frac{1}{\alpha}}\right)=\theta^{\frac{2}{\alpha}}$ for the path loss model.

In case of Rayleigh fading, the random variable $\Theta$ is multiplied by the random variable $W \triangleq \frac{G_{k}}{G_{\ell}}$ denoting the ratio of the two exponential distributed channel gains $G_{k}, G_{\ell}$. If $G_{k}, G_{\ell}$ are independent, then $W$ has distribution $F_{W}(w)=\frac{w}{w+1}$, where $0 \leq w \leq \infty$. The ratio $\Theta_{\text {Ray }}$ can then be calculated using the substitution $\Theta_{\text {Ray }}=W \Theta$ as

$$
\begin{aligned}
F_{\Theta_{\text {Ray }}}\left(\theta_{\text {Ray }}\right) & =\int_{0}^{1} \frac{\frac{\theta_{\text {Ray }}}{\theta}}{\frac{\theta_{\text {Ray }}}{\theta}+1} \frac{2}{\alpha} \theta^{\frac{2}{\alpha}-1} \mathrm{~d} \theta \\
& =\frac{2}{\alpha} \theta_{\text {Ray }} \int_{0}^{1} \frac{\theta^{\frac{2}{\alpha}-1}}{\theta_{\text {Ray }}+\theta} \mathrm{d} \theta \\
& =F\left(1, \frac{2}{\alpha}, \frac{2}{\alpha}+1, \frac{-1}{\theta_{\text {Ray }}}\right) .
\end{aligned}
$$

In the special case of $\alpha=4$, the cdf of $\Theta_{\text {Ray }}$ has the simple form $F_{\Theta_{\text {Ray }}}\left(\theta_{\text {Ray }}\right)=\sqrt{\theta_{\text {Ray }}} \arctan \left(1 / \sqrt{\theta_{\text {Ray }}}\right)$. 


\section{APPENDIX B}

ProOf OF THEOREM 2

The lower bound on $q(\lambda)$ : we neglect the second term on the right-hand side of (6) and restate the sufficient event $\left\{\tilde{\Pi}\left(\lambda_{M}\right) \backslash n_{1}(0) \cap d\left(0, r_{s}\right) \neq \emptyset\right\}$ as follows: outage occurs if at least one transmitter $X_{i} \neq n_{1}(0)$ lies within $d\left(0, r_{s}\right)$ and transmits in the same channel, according to (B.1). The event $\left\{\left(\Pi(\lambda) \backslash n_{1}(0)\right) \cap d\left(0, r_{s}\right) \neq \emptyset\right\}$ is the same as $\left\{\Pi(\lambda) \cap d\left(0, r_{s}\right) \geq 2\right\}$, i.e., there is more than one transmitter within $d\left(0, r_{s}\right)$. Given, there are $k$ transmitter in the near interference region, outage occurs if at least one node out of $\left\{n_{\ell}\left(X_{i}\right)\right\}_{\ell=2}^{k}$ transmits in the same channel. By construction this probability can be lower bounded as follows:

\section{$\mathbb{P}\left\{n_{\ell}\left(X_{i}^{\mathrm{rx}}\right)\right.$ creates outage $\}$}

$=\mathbb{P}\left\{X_{i}\right.$ is not nearest interferer of $\left.n_{\ell}^{\mathrm{rx}}\left(X_{i}^{\mathrm{rx}}\right)\right\} \frac{1}{M}$

$=\left(\mathbb{P}\left\{X_{i}\right.\right.$ not in scheduling range of $\left.n_{\ell}^{\mathrm{rx}}\left(X_{i}^{\mathrm{rx}}\right)\right\}$

$+\mathbb{P}\left\{X_{i}\right.$ in scheduling range but not nearest interferer $\left.\}\right) \frac{1}{M}$

$\geq \mathbb{P}\left\{X_{i}\right.$ not in scheduling range of $\left.n_{\ell}^{\mathrm{rx}}\left(X_{i}^{\mathrm{rx}}\right)\right\} \frac{1}{M}$,

where $n_{\ell}^{\mathrm{rx}}\left(X_{i}^{\mathrm{rx}}\right)$ is the associated receiver of the $\ell$-th nearest interferer of receiver $X_{i}^{\mathrm{rx}}$. The maximum separation distance of transmitter $X_{i}$ and $n_{\ell}^{\mathrm{rx}}\left(X_{i}^{\mathrm{rx}}\right)$ is $2 r+r \beta^{1 / \alpha}$ which follows from geometric arguments. Using the $\operatorname{CDF} F_{D}(d)=\frac{d^{2}}{(\max \{d\})^{2}}$ from [3] denoting the distance $d$ from an arbitrary point to the origin, (B.2) can be rewritten as

$$
\mathbb{P}\left\{n_{\ell}\left(X_{i}^{\mathrm{rx}}\right) \text { creates outage }\right\} \geq\left(1-\frac{\beta^{2 / \alpha}}{\left(2+\beta^{1 / \alpha}\right)^{2}}\right) \frac{1}{M} .
$$

Since outage occurs if at least one of the $\left\{n_{\ell}\left(X_{i}\right)\right\}_{\ell=2}^{k}$ nodes transmits in the same channel, we thus obtain

$\mathbb{P}\left\{\right.$ outage $\left.\mid \Pi(\lambda) \wedge d\left(0, r_{s}\right)=k\right\}=1-\left(1-\frac{4\left(1+\beta^{1 / \alpha}\right)}{M\left(2+\beta^{1 / \alpha}\right)^{2}}\right)^{k-1}$.

Applying the law of total probability yields the lower bound $q^{l}(\lambda)$.

The upper bound on $q(\lambda)$ is obtained by setting $\mathbb{P}\left\{n_{\ell}\left(X_{i}^{\mathrm{rx}}\right)\right.$ in the same channel as $\left.X_{i}\right\}=\frac{1}{M}$, i.e., assuming complete independence between receivers and nonorthogonalized interferers, and upper bounding the term $\mathbb{P}\left\{\tilde{Y}^{\mathrm{f}}>r^{-\alpha} \beta^{-1}\right\}$ in (6). In [5], the interference from the far interference region $\bar{d}\left(0, r_{s}\right)$ is upper bounded using the Chebychev's inequality $\mathbb{P}\{|X-\mathbb{E}\{X\}| \geq \kappa\} \leq \frac{\operatorname{Var}\{X\}}{\kappa}$ as:

$$
\mathbb{P}\left\{\tilde{Y}^{\mathrm{f}}>r^{-\alpha} \beta^{-1}\right\} \leq \frac{\lambda \pi r^{2} \beta^{2 / \alpha}}{M(\alpha-1)}\left(1-\frac{2 \pi \lambda r^{2} \beta^{2 / \alpha}}{M(\alpha-2)}\right)^{-2} .
$$

Substituting (B.5) in (6) finally yields the result.

\section{APPENDIX C \\ ProOF OF THEOREM 3}

The outage probability with a guard zone of radius $D$ around the receiver is given by [9] for the Rayleigh case:

$$
\begin{aligned}
q(\lambda \mid D & =d)=1-\exp \left\{\frac { \lambda \pi } { M } \left(d^{2}\left(1-\frac{1}{1+r^{\alpha} \beta d^{-\alpha}}\right)\right.\right. \\
& -r^{2} \beta^{\frac{2}{\alpha}} \Gamma\left(1-\frac{2}{\alpha}\right) \Gamma\left(1+\frac{2}{\alpha}\right) \\
& \left.\left.+r^{2} \beta^{\frac{2}{\alpha}} \mathbb{E}\left\{G^{\frac{2}{\alpha}} \Gamma\left(1-\frac{2}{\alpha}, G r^{\alpha} \beta d^{-\alpha}\right)\right\}\right)\right\} .
\end{aligned}
$$

Since the nearest interferer is orthogonalized, the radius $D$ of the guard zone is the same as $n_{1}(0)$, i.e., the distance from the nearest interferer to the reference receiver. To obtain $q(\lambda)$ from (C.1), one would thus need to average $q(\lambda \mid D=d)$ over all possible $D$, i.e., $q(\lambda)=\mathbb{E}\{q(\lambda \mid D)\}$ with respect to $D$. Since $q^{\prime}(\lambda \mid d)=0$ only for $d=0$ and $d \rightarrow+\infty$, and because $q(\lambda \mid d)$ is a differentiable decreasing function of $d$, it follows that $q(\lambda \mid d)$ has a unique inflection point $0<d_{s}<+\infty$. Thus, $q(\lambda \mid d)$ is strictly concave (convex) in $d$ for $d<d_{s}\left(d>d_{s}\right)$. Since $D$ is Rayleigh distributed, the expected distance $\mathbb{E}\{D\}$ to the nearest interferer is equal to $\frac{1}{2 \sqrt{\lambda}}$. The bounding effect of $q(\lambda)$ then follows directly from Jensen's inequality $\mathbb{E}\{f(X)\} \lesseqgtr f(\mathbb{E}\{X\})$ for a positive convex (concave) function $f$, when substituting $d=\mathbb{E}\{D\}$ in (C.1).

\section{REFERENCES}

[1] J. Andrews, S. Weber, and M. Haenggi, "Ad hoc networks: To spread or not to spread," IEEE Communications, Dec 2007.

[2] S. Weber, J. Andrews, X. Yang, and G. de Veciana, "Transmission capacity of wireless ad hoc networks with successive interference cancellation," IEEE Transactions on Information Theory, Aug 2007.

[3] O. B. S. Ali, C. Cardinal, and F. Gagnon, "On the performance of interference cancellation in wireless ad hoc networks," Trans. Comm., vol. 58, no. 2, pp. 433-437, 2010.

[4] N. Jindal, J. Andrews, and S. Weber, "Bandwidth partitioning in decentralized wireless networks," IEEE Transactions on Communications, Apr 2008

[5] S. Weber, J. Andrews, and N. Jindal, "An overview of the transmission capacity of wireless networks," IEEE Transactions on Communications, Jan 2010, under revision, http://arxiv.org/abs/0809.0016v4.

[6] S. Chakrabarti and A. Mishra, "Quality of service challenges for wireless mobile ad hoc networks: Research articles," Wirel. Commun. Mob. Comput., vol. 4, pp. 129-153, March 2004.

[7] D. Stoyan, W. Kendall, and J. Mecke, Stochastic geometry and its applications, 2nd ed. Wiley, 1995.

[8] J. Elsner, R. Tanbourgi, and F. Jondral, "On the Transmission Capacity of Wireless Multi-Channel Ad Hoc Networks with local FDMA scheduling," in International Congress on Ultra Modern Telecommunications and Control Systems, Oct 2010.

[9] J. Venkataraman, M. Haenggi, and O. Collins, "Shot noise models for outage and throughput analyses in wireless ad hoc networks," in Military Communications Conference, Oct 2006.

[10] D. J. Leith and P. Clifford, "Convergence of distributed learning algorithms for optimal wireless channel allocation," in in Proceedings of IEEE Conference on Decision and Control, 2006, pp. 2980-2985.

[11] M. Haenggi, "On distances in uniformly random networks," IEEE Transactions on Information Theory, Oct 2005.

$$
\left\{\left(\tilde{\Pi}\left(\lambda_{M}\right) \backslash n_{1}(0)\right) \cap d\left(0, r_{s}\right) \neq \emptyset\right\}=\left\{\left(\left(\Pi(\lambda) \backslash n_{1}(0)\right) \cap d\left(0, r_{s}\right) \neq \emptyset\right) \cap \text { (at least one interferer in the same channel) }\right\}
$$

